Supporting Information for:

\title{
Kalanchosides A-D, New Cytotoxic Bufadienolides from the Aerial Parts of Kalanchoe gracilis
}

Pei-Lin Wu, ${ }^{*}$ Yu-Lin Hsu, Tian-Shung Wu, K. F. Bastow, and Kuo-Hsiung Lee

wupl@mail.ncku.edu.tw 
S1.

\section{Table of Contents}

Table of Contents.......................... S2

Experimental Details..................... S3

Kalanchosides A-D (1-4) Data......... S4

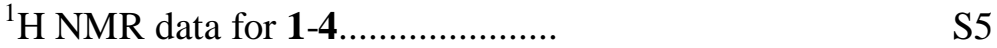

${ }^{13} \mathrm{C}$ NMR data for $1-4 \ldots \ldots \ldots \ldots \ldots \ldots \ldots \ldots \ldots$

${ }^{1}$ H NMR Spectrum of Kalanchoside A (1).................... S7

${ }^{13} \mathrm{C}$ NMR Spectrum of Kalanchoside A (1)................... S8

${ }^{1}$ H NMR Spectrum of Kalanchoside B (2).................... S9

${ }^{13}$ C NMR Spectrum of Kalanchoside B (2).................. S10

${ }^{1}$ H NMR Spectrum of Kalanchoside C (3).................... S11

${ }^{13}$ C NMR Spectrum of Kalanchoside C (3).................. S12

${ }^{1}$ H NMR Spectrum of Kalanchoside D (4)................... S13

${ }^{13}$ C NMR Spectrum of Kalanchoside D (4).................. S14 
S3.

Experimental Details:

General. Melting points were recorded on a Yanaco MP-3 melting point apparatus and are not corrected. Optical rotations were measured on a Jasco DIP-370 digital polarimeter. UV spectra were recorded on an Agilent 8453 spectrophotometer. IR spectra were recorded on a Nicolet Magna FT-IR spectrophotometer. NMR spectra were recorded on Bruker Avance 300 and AMX 400 FT-NMR spectrometers; all chemical shifts were given in ppm from tetramethylsilane as an internal standard. Mass spectra were obtained on a VG 70-250S spectrometer with a direct inlet system.

Plant Material. The aerial parts of Kalanchoe gracilis were purchased from a market (I-Shen galenic shop) in Taipei, Taiwan, in July 2003. It was authenticated by Professor C. S. Kuoh, Department of Biology, National Cheng Kung University, Tainan, Taiwan. A voucher specimen (No: PLW-0302) is deposited in the Herbarium of National Cheng Kung University, Tainan, Taiwan.

Extraction and Isolation. The dried aerial parts of $K$. gracilis $(5.5 \mathrm{~kg})$ were extracted with $\mathrm{MeOH}$ under reflux $(8 \times)$. The combined extracts were concentrated under reduced pressure to give a dark brown syrup. The syrup was then suspended in $\mathrm{H}_{2} \mathrm{O}$, defatted with hexane and then partitioned with $\mathrm{CHCl}_{3}$ and EtOAc, successively. The concentrated $\mathrm{CHCl}_{3}$ layer $(14 \mathrm{~g})$ was chromatographed on a silica gel column, eluting with a gradient of hexane- $\mathrm{Me}_{2} \mathrm{CO}(4: 1)$ to pure $\mathrm{Me}_{2} \mathrm{CO}$ to yield ten fractions. Fraction 6 was subjected to repeated chromatography on silica gel to give 7 (5 mg). Fraction 7 was repeatedly chromatographed on a silica gel column to give 4 (10 $\mathrm{mg}), 6$ (25 mg), and 8 (19 mg), successively. Fraction 8 was rechromatographed to give $\mathbf{3}$ (9 mg). The concentrated EtOAc layer (50 g) was subjected to column chromatography on Cosmosil 75 $\mathrm{C} 18$, eluted with a gradient of $\mathrm{H}_{2} \mathrm{O}-\mathrm{MeOH}$ (from pure $\mathrm{H}_{2} \mathrm{O}$ to pure $\mathrm{MeOH}$ ) to give nine fractions. Fraction 2 was subjected to chromatography on a silica gel column eluting with a gradient of $\mathrm{CHCl}_{3}-\mathrm{Me}_{2} \mathrm{CO}(1: 2)$ to pure $\mathrm{Me}_{2} \mathrm{CO}$ to give 1 (14 mg) and 2 (3 mg). Fraction 3 was further chromatographed on a silica gel column eluting with a gradient of $\mathrm{CHCl}_{3}-\mathrm{MeOH}(5: 1)$ to pure $\mathrm{MeOH}$ to give $\mathbf{5}(8 \mathrm{mg})$. 
S4. Kalanchosides A-D (1-4) Data

Kalanchoside A (1). White amorphous powder; $[\alpha]_{\mathrm{D}}-41.9^{\circ}$ (c $\left.0.11, \mathrm{MeOH}\right)$; UV $(\mathrm{MeOH})$ $\lambda_{\max }(\log \varepsilon) 205$ (4.0), $214(3.9, \mathrm{sh}), 295$ (3.2) nm; IR (KBr) $v_{\max } 3402,1709,1529 \mathrm{~cm}^{-1}$; FABMS $m / z 585[\mathrm{M}+\mathrm{Na}]^{+}$; HR-FABMS $m / z 563.2853[\mathrm{M}+\mathrm{H}]^{+}\left(\right.$calcd for $\mathrm{C}_{30} \mathrm{H}_{43} \mathrm{O}_{10}$ 563.2856).

${ }^{1} \mathrm{H}-\mathrm{NMR}$ data, Table $1 ;{ }^{13} \mathrm{C}-\mathrm{NMR}$ data, Table 2.

Kalanchoside B (2). White amorphous powder; $[\alpha]_{\mathrm{D}}-48.4^{\circ}(\mathrm{c} 0.12, \mathrm{MeOH})$; UV $(\mathrm{MeOH})$ $\lambda_{\max }(\log \varepsilon) 203$ (3.6), $222(3.2, \mathrm{sh}), 297(2.8) \mathrm{nm}$; IR (KBr) $v_{\max } 3418,1713,1538 \mathrm{~cm}^{-1}$; FABMS $m / z 585[\mathrm{M}+\mathrm{Na}]^{+}, 563[\mathrm{M}+\mathrm{H}]^{+} ;$HR-FABMS $m / z$. $563.2855[\mathrm{M}+\mathrm{H}]^{+}\left(\right.$calcd for $\mathrm{C}_{30} \mathrm{H}_{43} \mathrm{O}_{10}$ 563.2856). ${ }^{1} \mathrm{H}-\mathrm{NMR}$ data, Table $1 ;{ }^{13} \mathrm{C}-\mathrm{NMR}$ data, Table 2.

Kalanchoside C (3). White amorphous powder; $[\alpha]_{\mathrm{D}}-9.6^{\circ}(\mathrm{c} 0.23, \mathrm{MeOH}) ; \mathrm{UV}(\mathrm{MeOH})$ $\lambda_{\max }(\log \varepsilon) 207(3.7), 296(2.9) \mathrm{nm} ; \mathrm{IR}(\mathrm{KBr}) v_{\max } 3382,1704,1538 \mathrm{~cm}^{-1} ;$ FABMS m/z $561[\mathrm{M}+$ $\mathrm{H}]^{+}$; HR-FABMS $m / z$ 561.2702 [M + H $]^{+}$(calcd for $\mathrm{C}_{30} \mathrm{H}_{41} \mathrm{O}_{10}$ 561.2700). ${ }^{1} \mathrm{H}-\mathrm{NMR}$ data, Table 1; ${ }^{13} \mathrm{C}-\mathrm{NMR}$ data, Table 2.

Kalanchoside D (4). White amorphous powder; $[\alpha]_{\mathrm{D}}-107.6^{\circ}$ (c 0.49, MeOH); UV (MeOH) $\lambda_{\max }(\log \varepsilon) 208(3.4), 262(2.7), 296(2.5) \mathrm{nm} ; \mathrm{IR}(\mathrm{KBr}) v_{\max } 3365,1709,1538 \mathrm{~cm}^{-1}$; FABMS $\mathrm{m} / z$ $491[\mathrm{M}+\mathrm{H}]^{+}$; HR-FABMS m/z 491.2278 $[\mathrm{M}+\mathrm{H}]^{+}$(calcd for $\left.\mathrm{C}_{26} \mathrm{H}_{35} \mathrm{O}_{9} 491.2281\right) .{ }^{1} \mathrm{H}-\mathrm{NMR}$ data, Table $1 ;{ }^{13} \mathrm{C}-\mathrm{NMR}$ data, Table 2. 
S5. Table 1. ${ }^{1} \mathrm{H}$ NMR data for $\mathbf{1 - 4}{ }^{\mathrm{a}}$

\begin{tabular}{|c|c|c|c|c|}
\hline position & 1 & 2 & 3 & 4 \\
\hline $\mathrm{H}-1$ & $1.66 ; 2.17$ & $1.66 ; 2.14$ & $1.65 ; 2.29$ & $4.65 \mathrm{dd}(12.6,3.7)$ \\
\hline $\mathrm{H}-2$ & 1.78 & 1.72 & $1.76 ; 1.94$ & $1.50(\alpha) ; 2.07(\beta)$ \\
\hline $\mathrm{H}-3$ & 4.07 br s & 4.05 br s & 4.03 br s & 3.81 br s \\
\hline $\mathrm{H}-4$ & $1.58 ; 2.10$ & $1.60 ; 2.07$ & $1.71 ; 2.06$ & 1.89 \\
\hline H-6 & $1.61 ; 2.17$ & $1.62 ; 2.18$ & $1.71 ; 2.19$ & $1.50(\beta) ; 1.95(\alpha)$ \\
\hline $\mathrm{H}-7$ & $1.39 ; 2.25$ & $1.34 ; 2.20$ & $1.42 ; 2.29$ & $1.18(\alpha) ; 1.84(\beta)$ \\
\hline H-8 & 1.96 & $1.95 \operatorname{td}(12.4,3.7)$ & $2.46 \operatorname{td}(12.6,2.8)$ & $2.50 \operatorname{td}(11.5,2.5)$ \\
\hline H-9 & 1.78 & $1.75 \operatorname{td}(12.4,3.0)$ & 1.96 & $1.29 \mathrm{t}(11.5)$ \\
\hline \multirow[t]{2}{*}{$\mathrm{H}-11$} & $1.16 ; 1.58$ & $1.16 ; 1.50$ & $2.27 \mathrm{dd}(14.0,4.2)$ & $3.99 \operatorname{td}(11.5,2.6)$ \\
\hline & & & $2.59 \mathrm{t}(14.0)$ & \\
\hline $\mathrm{H}-12$ & $1.35 ; 1.45$ & $1.40 ; 1.48$ & & $1.46(\alpha) ; 2.04(\beta)$ \\
\hline $\mathrm{H}-15$ & $1.64 ; 2.10$ & $1.66 ; 2.10$ & $1.42 ; 1.71$ & $1.56(\beta) ; 2.14(\alpha)$ \\
\hline H-16 & $1.76 ; 2.07$ & $1.69 ; 2.07$ & $1.71 ; 1.90$ & $1.84(\beta) ; 2.18(\alpha)$ \\
\hline $\mathrm{H}-17$ & $2.54 \mathrm{dd}(9.0,6.5)$ & $2.53 \mathrm{dd}(9.7,6.0)$ & $3.95 \mathrm{~m}$ & $2.69 \mathrm{t}(8.1)$ \\
\hline H-18 & $0.69 \mathrm{~s}$ & $0.69 \mathrm{~s}$ & $0.88 \mathrm{~s}$ & $0.86 \mathrm{~s}$ \\
\hline H-19 & $10.07 \mathrm{~s}$ & $10.07 \mathrm{~s}$ & $10.05 \mathrm{~s}$ & $5.79 \mathrm{~d}(4.8)$ \\
\hline $\mathrm{H}-21$ & $7.40 \mathrm{~d}(1.6)$ & $7.39 \mathrm{~d}(2.2)$ & $7.44 \mathrm{~d}(1.5)$ & $7.41 \mathrm{~d}(2.5)$ \\
\hline H-22 & $7.95 \mathrm{dd}(9.8,1.6)$ & $7.95 \mathrm{dd}(9.7,2.2)$ & $7.85 \mathrm{dd}(9.8,1.5)$ & $7.83 \mathrm{dd}(9.7,2.5)$ \\
\hline $\mathrm{H}-23$ & $6.17 \mathrm{~d}(9.8)$ & $6.17 \mathrm{~d}(9.7)$ & $6.20 \mathrm{~d}(9.8)$ & $6.20 \mathrm{~d}(9.7)$ \\
\hline H-1' & $4.87 \mathrm{~d}(3.7)$ & $4.88 \mathrm{~d}(3.2)$ & $4.85 \mathrm{~d}(3.9)$ & \\
\hline H-2' & $3.44 \mathrm{dd}(9.3,3.7)$ & $3.76 \mathrm{~m}$ & $3.53 \mathrm{dt}(8.7,3.9)$ & \\
\hline H-3' & 3.60 t (9.3) & $3.76 \mathrm{~m}$ & $3.95 \mathrm{~m}$ & \\
\hline H-4' & 2.99 t (9.3) & 3.68 br s & $1.51(\mathrm{ax}), 1.83(\mathrm{eq}) \mathrm{m}$ & \\
\hline H-5' & $3.63 \mathrm{dq}(9.3,6.3)$ & 3.97 br q (6.4) & $4.25 \mathrm{~m}$ & \\
\hline H-6' & $1.18 \mathrm{~d}(6.3)$ & $1.18 \mathrm{~d}(6.4)$ & $1.11 \mathrm{~d}(6.3)$ & \\
\hline $\mathrm{OH}-2^{\prime}$ & $3.62 \mathrm{brs}$ & 3.57 br s & $3.88 \mathrm{~d}(8.7)$ & \\
\hline $\mathrm{OH}-3^{\prime}$ & $-{ }^{b}$ & $-\mathrm{b}$ & $3.74 \mathrm{~d}(5.4)$ & \\
\hline OH-4' & 4.08 br s & $-{ }^{b}$ & & \\
\hline OH-3 & & & & $3.94 \mathrm{~s}$ \\
\hline OH-5 & $4.68 \mathrm{~s}$ & $4.70 \mathrm{~s}$ & $4.81 \mathrm{~s}$ & $5.03 \mathrm{~s}$ \\
\hline $\mathrm{OH}-14$ & $3.43 \mathrm{~s}$ & $3.45 \mathrm{~s}$ & $4.15 \mathrm{~s}$ & $3.52 \mathrm{~s}$ \\
\hline OH-19 & & & & $6.59 \mathrm{~s}$ \\
\hline OAc & & & & $2.02 \mathrm{~s}$ \\
\hline
\end{tabular}

${ }^{a} \delta$, multiplicity, $J, \mathrm{~Hz}$ in parentheses, all compounds measured in acetone- $\mathrm{d}_{6}$

${ }^{b}$ The chemical shift of $\mathrm{OH}$ may overlap with those of sugar's protons 
S6. Table $2 .{ }^{13} \mathrm{C}$ NMR data for $\mathbf{1}-\mathbf{4}^{\mathrm{a}}$

\begin{tabular}{|c|c|c|c|c|}
\hline Position & 1 & 2 & 3 & 4 \\
\hline C-1 & 18.8 & 18.9 & 19.2 & 73.0 \\
\hline C-2 & 26.2 & 26.1 & 25.7 & 39.5 \\
\hline C-3 & 72.1 & 71.9 & 75.7 & 65.3 \\
\hline C-4 & 37.9 & 38.0 & 37.8 & 47.6 \\
\hline C-5 & 73.5 & 73.5 & 73.8 & 76.2 \\
\hline C-6 & 34.7 & 34.7 & 35.8 & 35.1 \\
\hline C-7 & 25.1 & 25.2 & 25.2 & 20.5 \\
\hline C-8 & 42.8 & 42.8 & 41.7 & 39.7 \\
\hline C-9 & 40.0 & 40.0 & 36.6 & 49.5 \\
\hline C-10 & 55.7 & 55.7 & 55.0 & 54.5 \\
\hline C-11 & 23.2 & 23.2 & 38.3 & 79.9 \\
\hline C-12 & 41.0 & 41.1 & 211.8 & 47.9 \\
\hline C-13 & 48.9 & 48.9 & 64.0 & 52.7 \\
\hline C-14 & 84.9 & 85.0 & 85.6 & 86.0 \\
\hline C-15 & 32.3 & 32.4 & 32.2 & 32.2 \\
\hline C-16 & 29.3 & 29.3 & 29.5 & 31.0 \\
\hline C-17 & 51.6 & 51.6 & 40.6 & 51.4 \\
\hline C-18 & 16.9 & 16.8 & 17.4 & 20.5 \\
\hline C-19 & 208.5 & 208.5 & 209.1 & 104.8 \\
\hline C-20 & 123.4 & 123.4 & 122.1 & 122.8 \\
\hline C-21 & 150.0 & 150.0 & 150.9 & 149.9 \\
\hline C-22 & 147.8 & 147.7 & 147.7 & 147.5 \\
\hline C-23 & 115.3 & 115.4 & 115.7 & 115.5 \\
\hline C-24 & 162.0 & 162.0 & 162.0 & 161.9 \\
\hline C-1' & 97.3 & 97.7 & 100.7 & \\
\hline C-2' & 73.0 & 69.3 & 68.7 & \\
\hline C-3' & 74.7 & 71.2 & 68.3 & \\
\hline C-4' & 76.9 & 72.8 & 40.4 & \\
\hline C-5' & 69.0 & 67.5 & 60.3 & \\
\hline C-6' & 18.2 & 16.9 & 21.0 & \\
\hline OAc & & & & $21.0,170.9$ \\
\hline
\end{tabular}

${ }^{a}$ All compounds measured in acetone- $\mathrm{d}_{6}$ 
S7. ${ }^{1} \mathrm{H}$ NMR spectrum of kalanchoside A (1) in acetone- $\mathrm{d}_{6}$
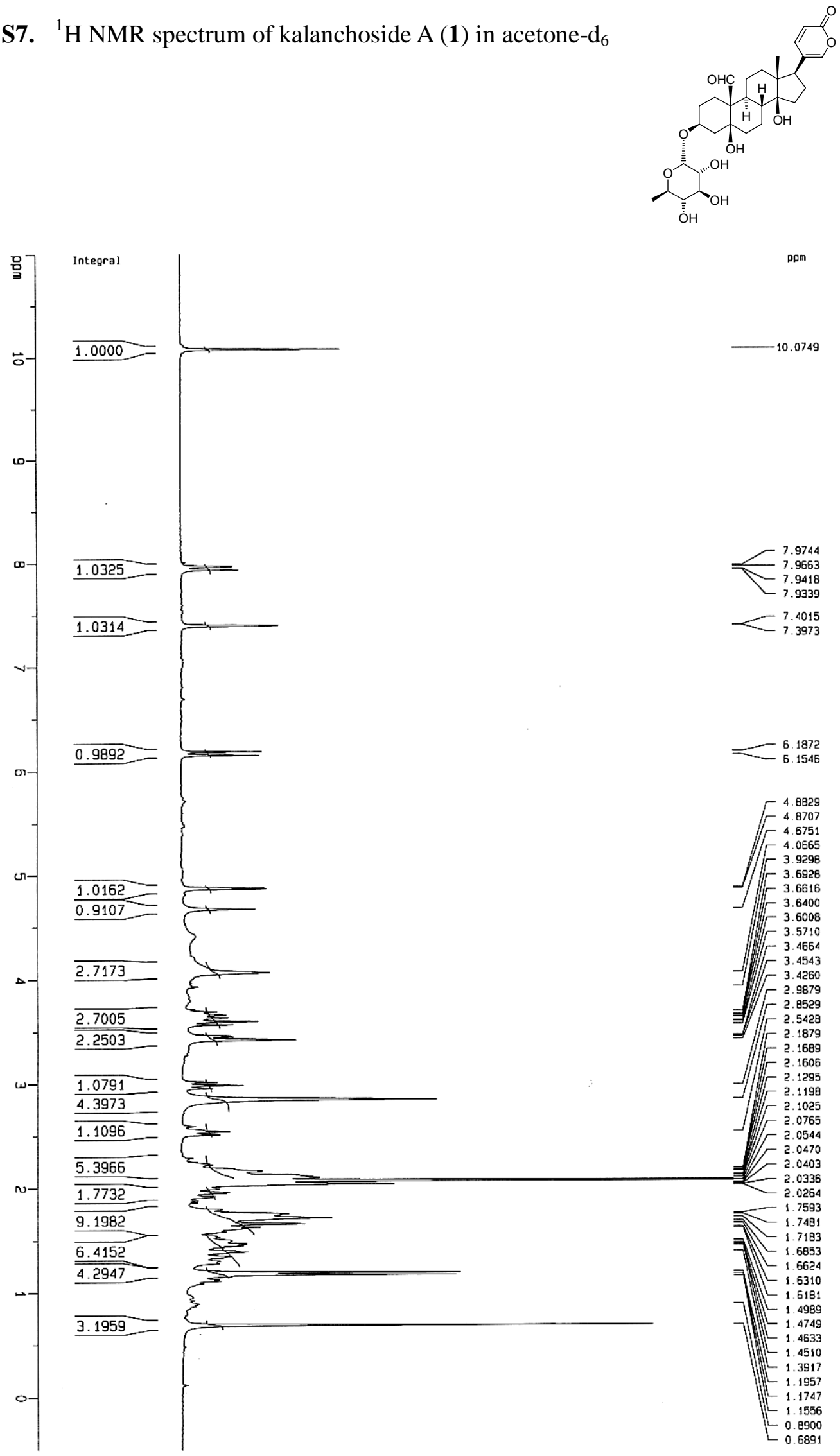
S18. ${ }^{13} \mathrm{C}$ NMR spectrum of kalanchoside A (1) in acetone- $\mathrm{d}_{6}$
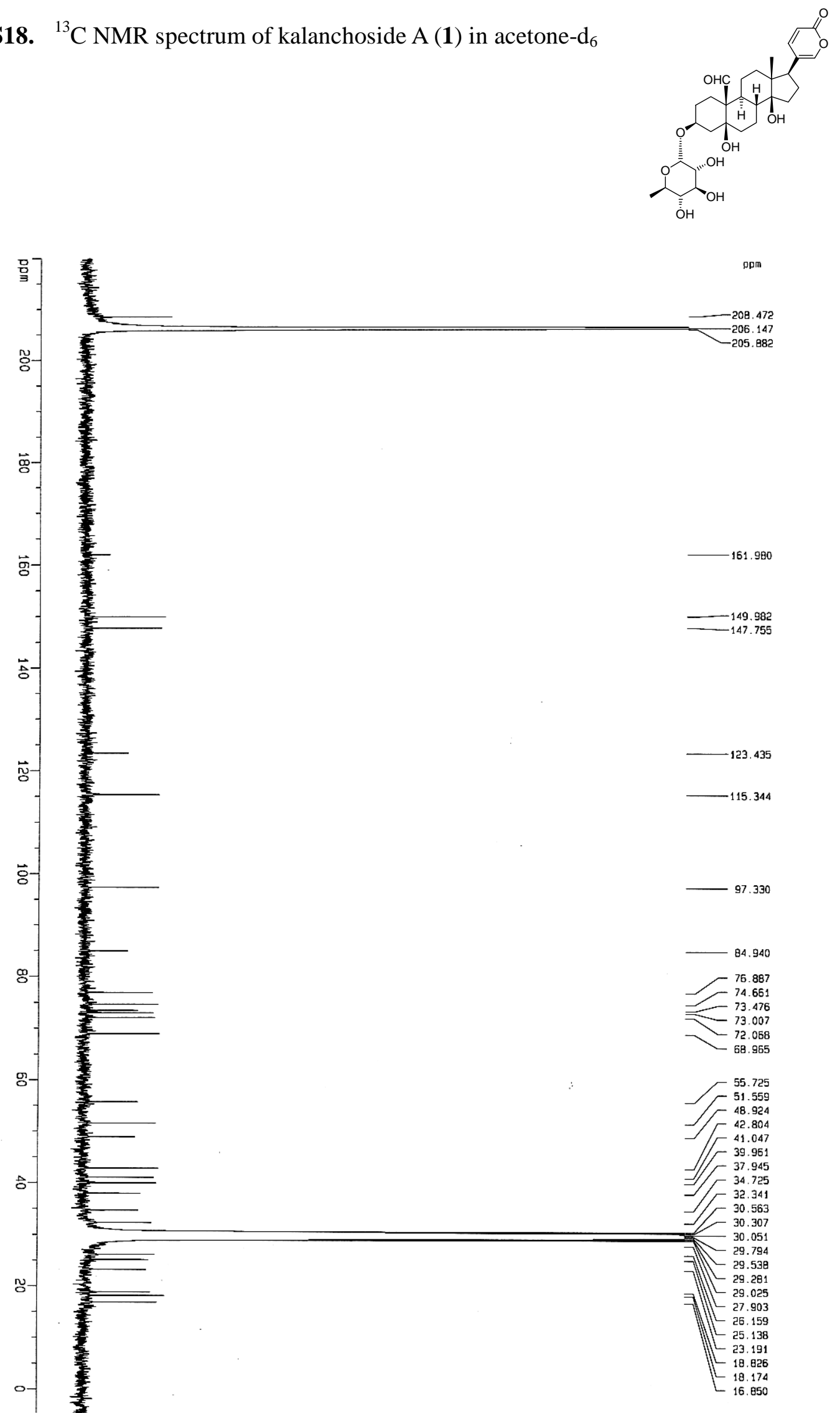
S19. ${ }^{1} \mathrm{H}$ NMR spectrum of kalanchoside B (2) in acetone- $\mathrm{d}_{6}$

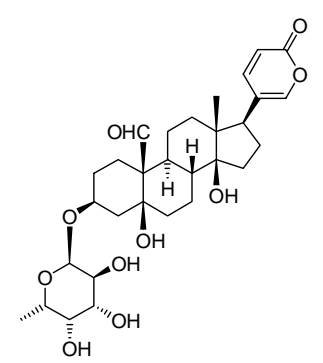

읍

Integral

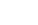

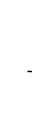

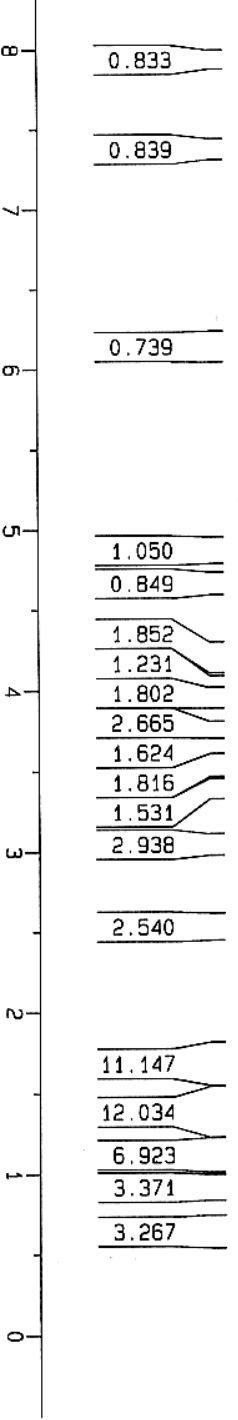

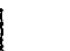

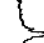

$0.854=$

0.854
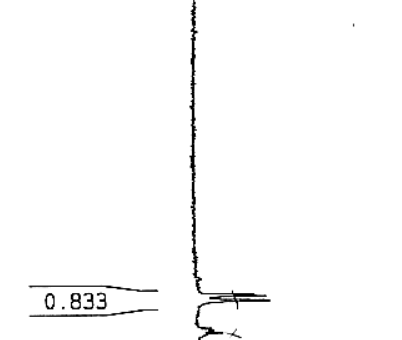

$\xi$
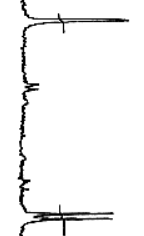

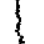
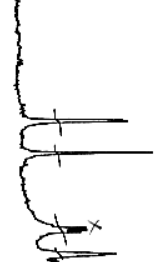

핟
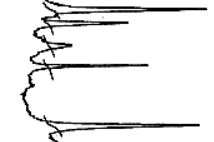

$-10.0704$

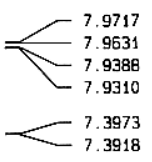

$=6.1845$ 6.1521 4.8878 $-4.8803$

4.6837

- 4.2084 - 4.0534 3.9880 IIF $\begin{array}{r}3.9664 \\ 3.9461\end{array}$ - $\begin{array}{r}3.8434 \\ -3.7637\end{array}$ 3.7637 - 3.6813 $-3.4177$ - 2.5431 - $\begin{array}{r}2.5120 \\ 2.1602\end{array}$ 2.1602
-2.1325 $\begin{array}{r}2.1325 \\ -2.0970 \\ \hline\end{array}$ 2.0970
-2.0769 Dr 2.0769 NF 2.0478 AfF $\begin{array}{r}2.0405 \\ 2.0334\end{array}$ If 2.0264 $-1.7460$ 1.6581 $7-1.6492$ $\exists-1.6295$ $7\left[\begin{array}{l}1.4747 \\ -1.4629\end{array}\right.$ $7\left[\begin{array}{l}1.4503 \\ 1.3929\end{array}\right.$

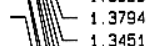
站 1.3059 非 1.2803 , 1.1722 $\left[\begin{array}{l}1.1722 \\ -0.9285\end{array}\right.$ L 0.8922 
S10. ${ }^{13} \mathrm{C}$ NMR spectrum of kalanchoside B (2) in acetone- $\mathrm{d}_{6}$

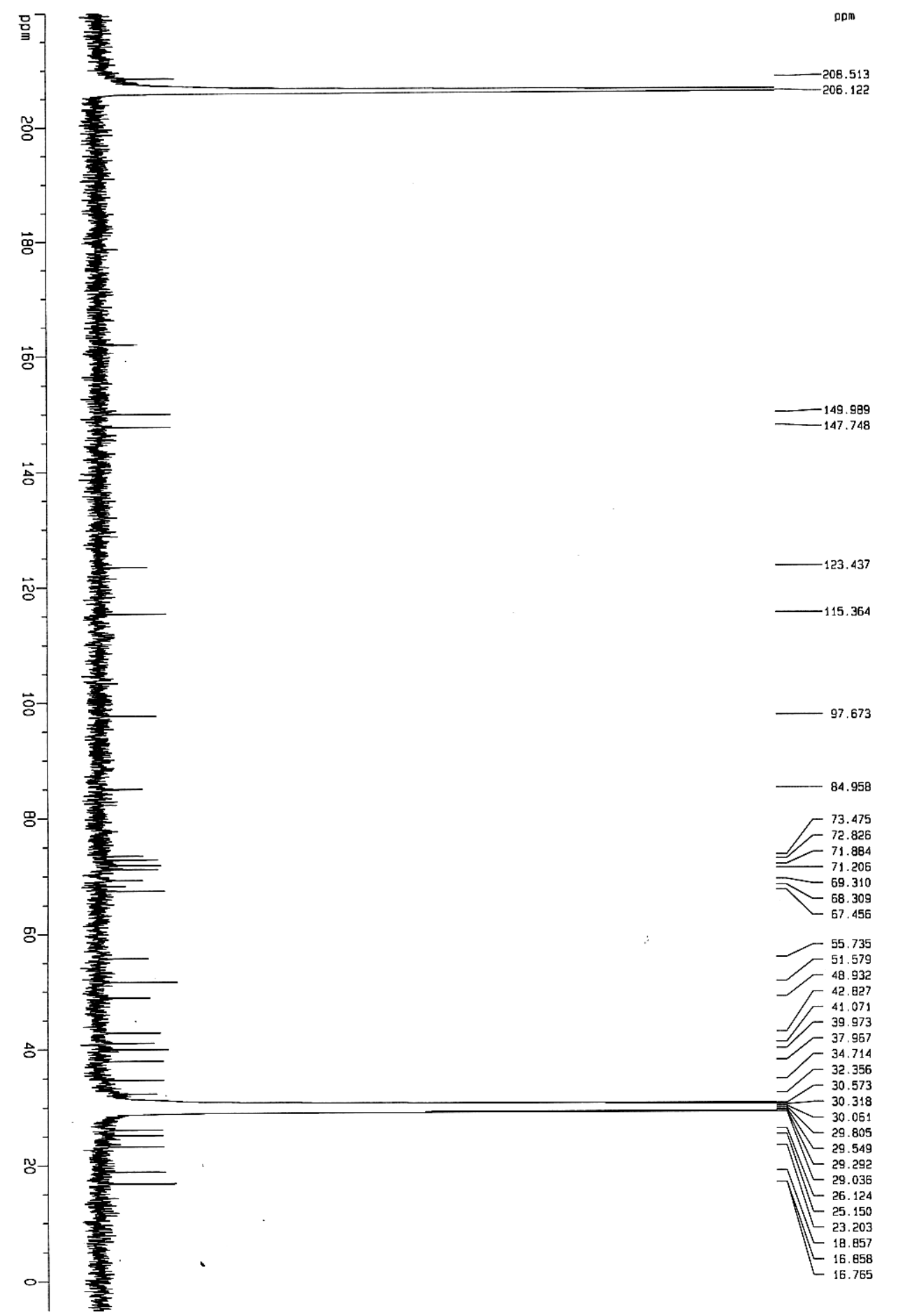


S11. ${ }^{1} \mathrm{H}$ NMR spectrum of kalanchoside $\mathrm{C}(3)$ in acetone- $\mathrm{d}_{6}$
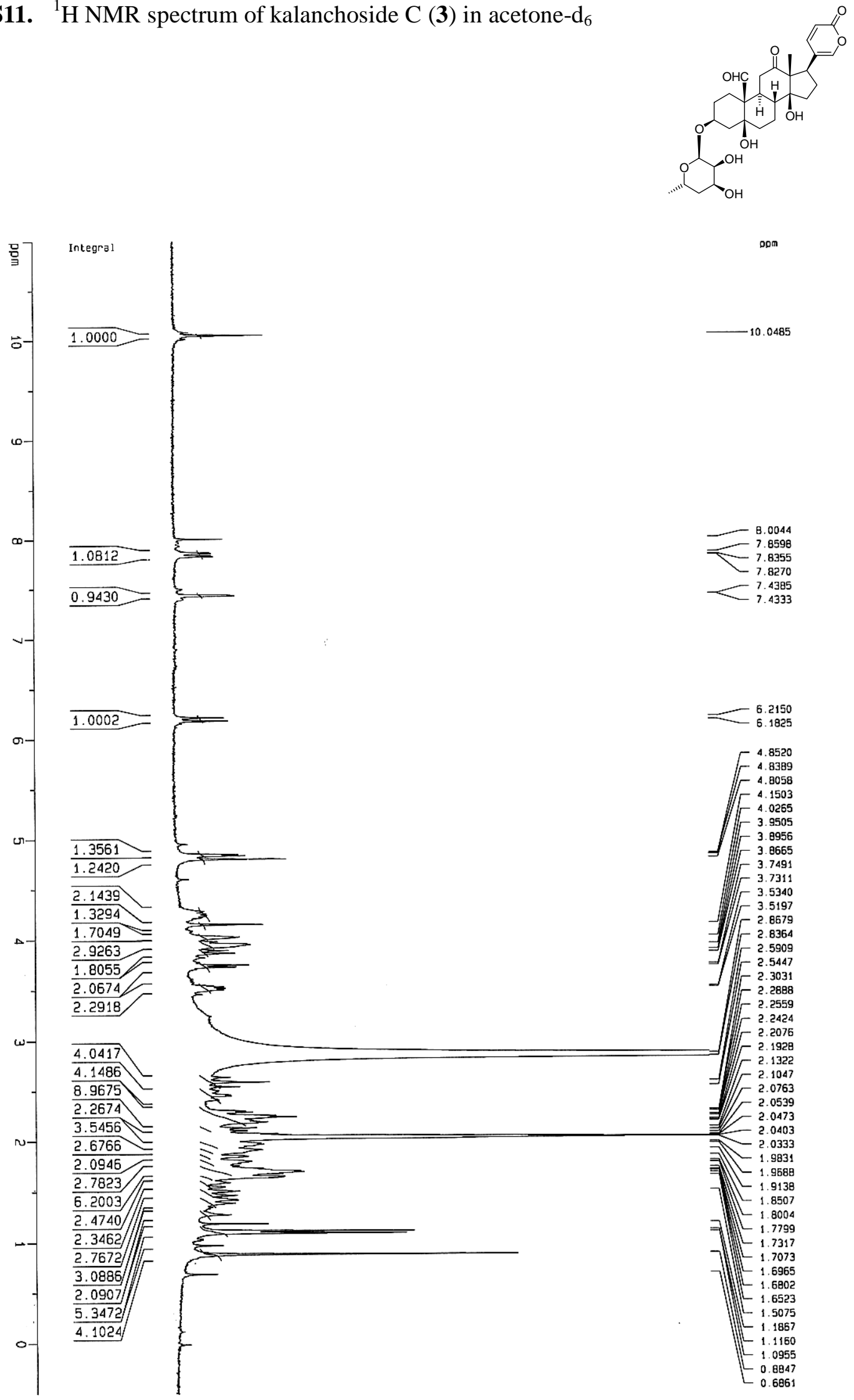
S12. ${ }^{13} \mathrm{C}$ NMR spectrum of kalanchoside $\mathrm{C}(\mathbf{3})$ in acetone- $\mathrm{d}_{6}$
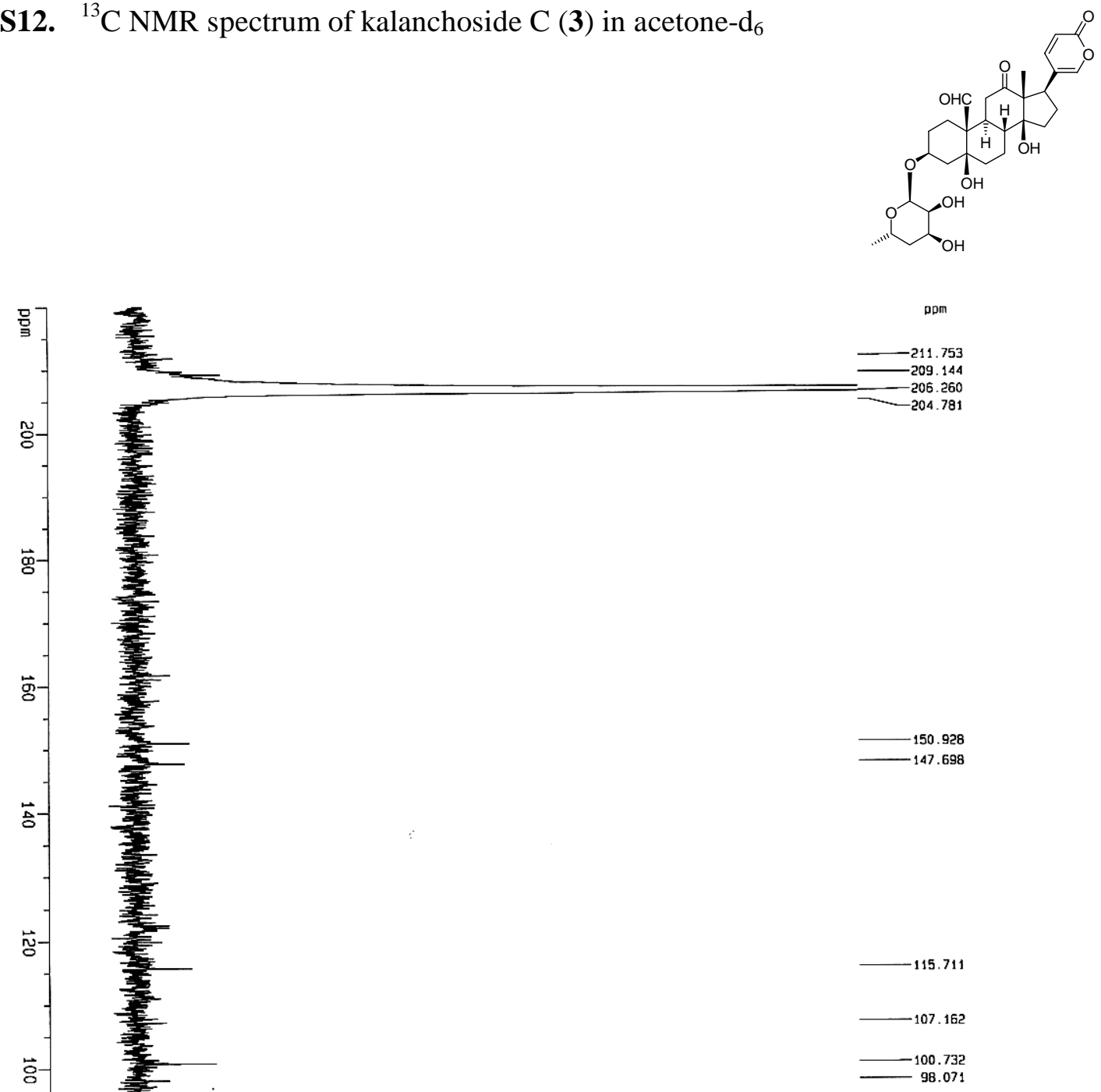

g

o

บั-

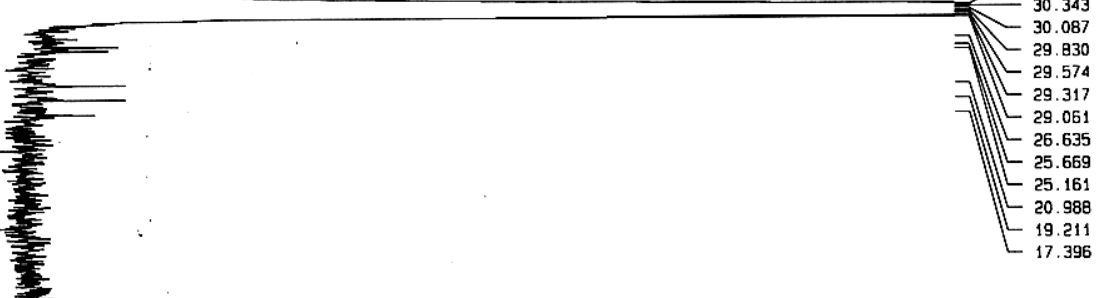

o-

\begin{tabular}{ll}
17.396 \\
\hline
\end{tabular} 
S13. ${ }^{1} \mathrm{H}$ NMR spectrum of kalanchoside D (4) in acetone- $\mathrm{d}_{6}$
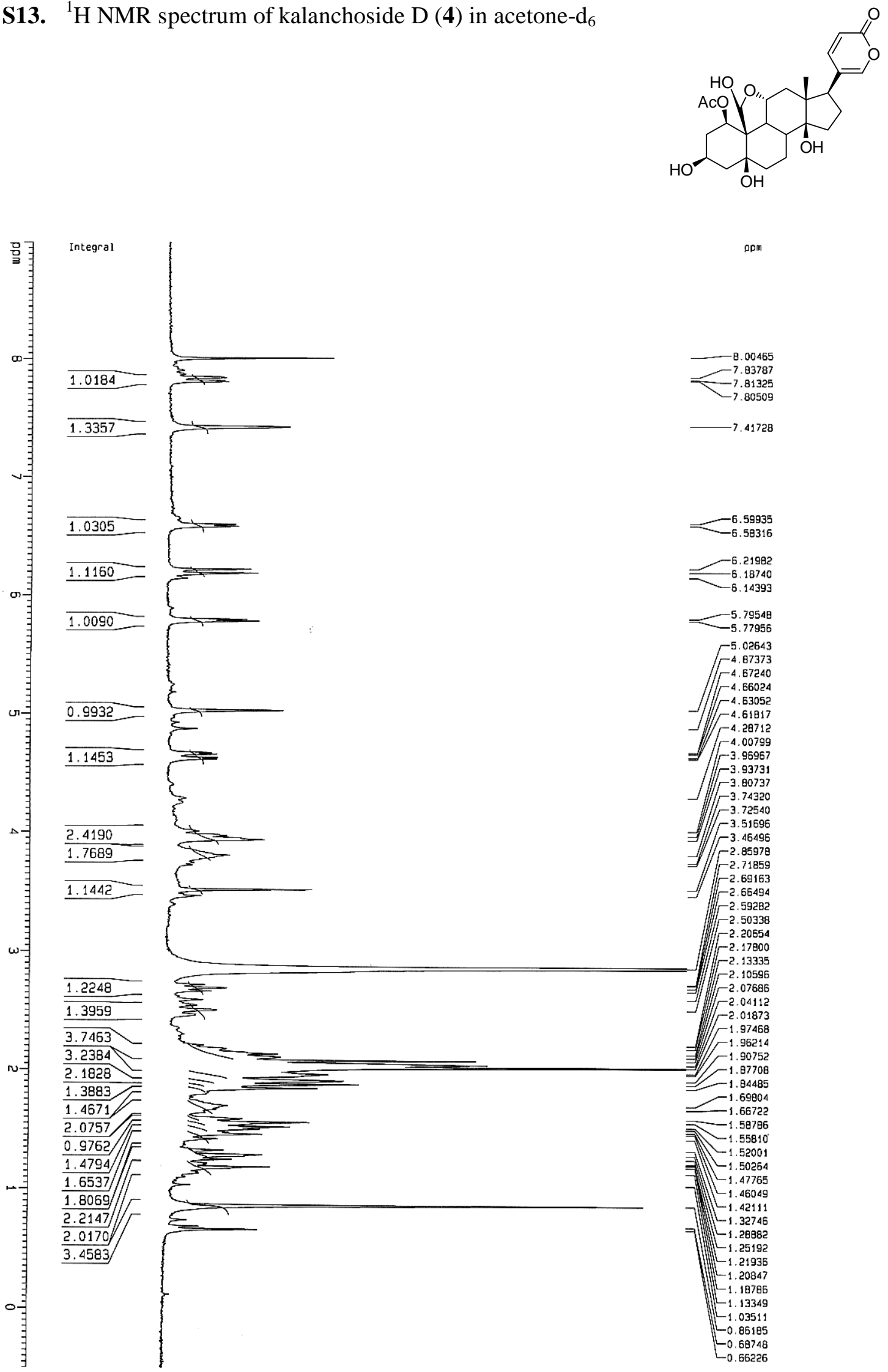
S14. ${ }^{13} \mathrm{C}$ NMR spectrum of kalanchoside $\mathrm{D}(4)$ in acetone- $\mathrm{d}_{6}$

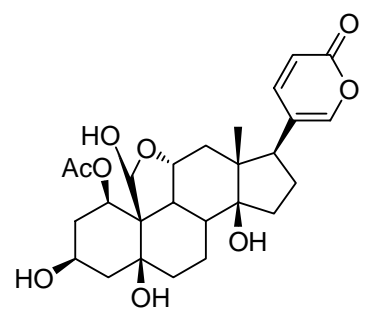

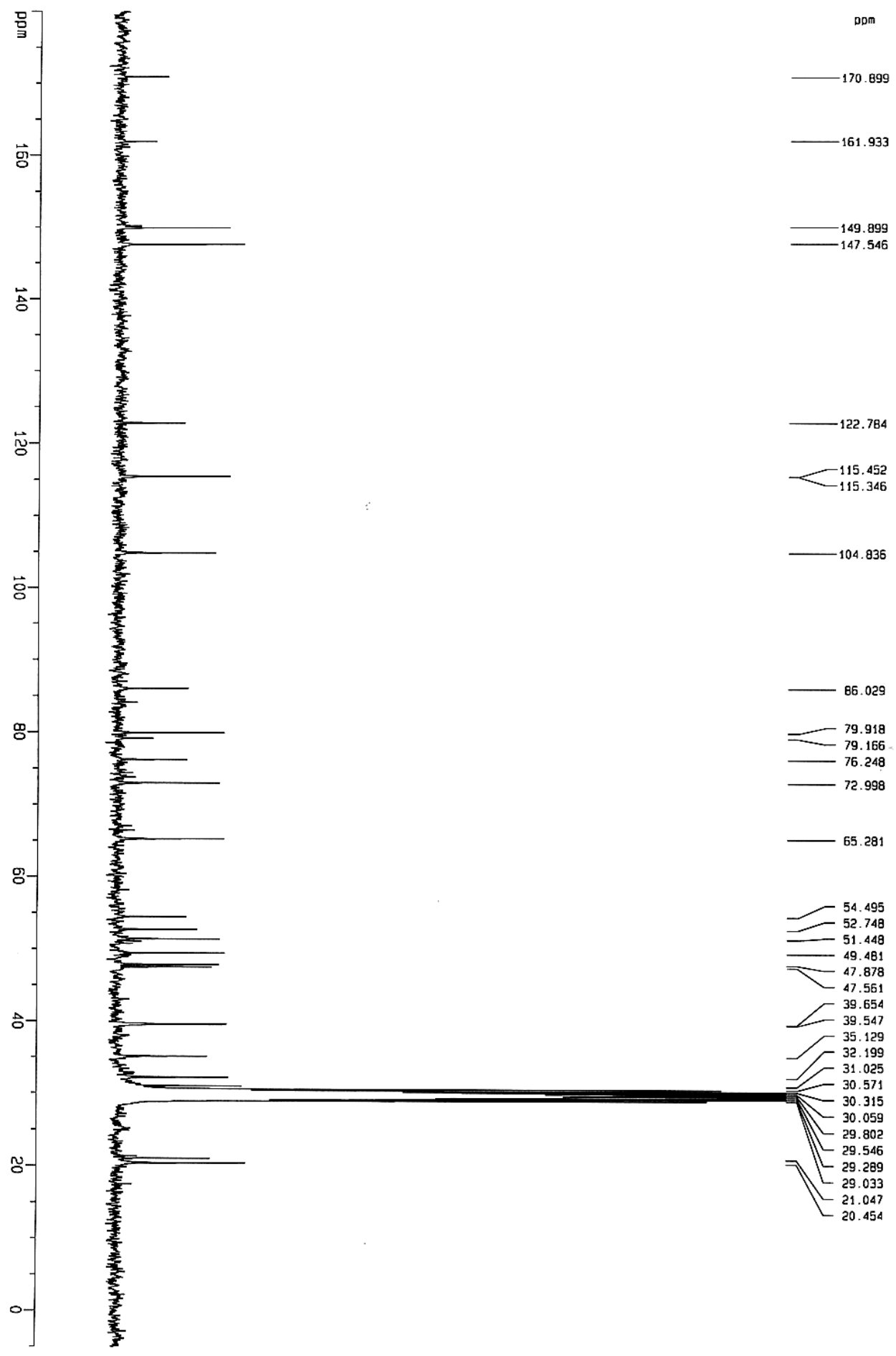

